\title{
Editorial
}

\section{MORTALIDAD PERINATAL EN LAS AMÉRICAS}

La tasa de mortalidad perinatal es un indicador que resume el riesgo de morir del feto y recién nacido como consecuencia del proceso reproductivo. Contribuyen además los factores ambientales y el grado de desarrollo social, económico y sanitario de un país.

El Centro de Perinatología y Desarrollo Humano (CLAP) (1) nos presenta en su página web, la estadística de la mortalidad perinatal en la Región de las Américas, actualizada a octubre de 2003. En ella podemos observar las enormes diferencias que se establecen entre los países desarrollados y en desarrollo de la Región. Es así que la tasa de mortalidad perinatal promedio de Canadá y Estados Unidos de Norteamérica es de 7/ 1000 nacidos vivos, mientras que en Sudamérica es de 21/1000 nacidos vivos, en Centroamérica de $35 / 1000$ nacidos vivos, en el Caribe no latino de $34 / 1000$ nacidos vivos y en el Caribe latino de $53 / 1000$ nacidos vivos. Cifras directamente relacionadas con el grado de desarrollo económico, social y sanitario de esas regiones. En la tabla siguiente se presenta el indicador analizado correspondiente a los países sudamericanos, proporcionado por la misma fuente. Cuba, país líder en Latino América en múltiples indicadores de salud tiene una tasa de mortalidad perinatal de $12,6 / 1000$ nacidos vivos (Tabla 1$)(1,2)$.

Chile presenta una mortalidad perinatal muy cercana a la de los países desarrollados de la Región, producto de las políticas de salud de los diferentes gobiernos para reducir las tasas de mortalidad dependientes del proceso reproductivo, implementando políticas preventivas como el control de la fecundidad, la amplia cobertura del control prenatal y de la atención profesional del parto, la alimentación suplementaria de la embarazada, la fortificación de las harinas con ácido fólico, y desde el $1^{\circ}$ de julio de 2005 (3) la garantía del tratamiento de las disrrafias espinales, el tratamiento quirúrgico de las cardiopatías congénitas y la prevención del parto prematuro y de sus complicaciones, que deberían reflejarse en el descenso de las tasas de mortalidad perinatal e infantil de los próximos años, y así también lograr disminuir las brechas de desigualdad de esos indicadores entre los diferentes sectores sociales del país (4, 5). Chile, especialmente desde 1990 presenta una significativa reducción de las tasas de mortalidad fetal tardía (6) y neonatal precoz (7) gracias a las políticas de salud reproductiva antes señaladas, sin recurrir a la legalización del aborto inducido, estrategia utilizada por los países desarrollados para el control de esos indicadores (8).

En este número de la Revista Chilena de Obstetricia y Ginecología, el Dr Manuel Ticona y equipo, nos privilegia al presentar los resultados de su investigación titulada "MORTALIDAD PERINATAL HOSPITALARIA EN EL PERÚ: FACTORES DE RIESGO" (9). Como pudimos observar en la tabla anterior, Perú tiene una mortalidad perinatal que necesariamente obliga al país hermano a fortalecer las políticas de salud reproductiva que permitan disminuir esa tasa. Ticona identifica claramente los factores de riesgo de

Tabla 1

\begin{tabular}{lcr}
\hline Países & $\begin{array}{c}\text { Mortalidad perinatal } \\
\text { (x 1000 nacidos vivos) } \\
\text { Tasa }\end{array}$ \\
\hline Argentina & 16,3 & $n$ \\
Bolivia & 55,0 & 11.194 \\
Brasil & 22,5 & 14.030 \\
Chile & 8,8 & 78.093 \\
Colombia & 24,0 & 2.512 \\
Ecuador & 45,0 & 23.357 \\
Paraguay & 40,0 & 13.266 \\
Perú & 23,0 & 6.976 \\
Uruguay & 16,5 & 14.382 \\
Venezuela & 23,7 & 942 \\
\hline
\end{tabular}

Fuente: www.clap.ops-oms.org 
mayor importancia (prematurez, bajo peso al nacer, asfixia perinatal y multiparidad) sobre los cuales hay que intervenir para lograr una reducción significativa del indicador. Esperamos que Perú pueda implementar políticas destinadas a ese fin considerando el diagnóstico efectuado.

\section{Dr. ENRIQUe Donoso Siña Editor Jefe}

\section{BIBLIOGRAFÍA}

1. Centro Latino Americano de Perinatología y Desarrollo Humano (Clap OPS/OMS). Estadísticas de la Región de América Latina y Caribe. Hallado en: www.clap.ops-oms.org

2. Donoso E, Donoso A, Villarroel L. Mortalidad perinatal e infantil y el bajo peso al nacer en Chile y Cuba en el período 1991-2001. ReV ChIL OBStet GINECOL 2004; 69(3): 203-208.
3. Ministerio de Salud, Gobierno de Chile. Ley 19.996: Régimen general de garantías en salud. REV CHIL OBSTET GINECOL 2005; 70(2): 119-29.

4. Donoso E. Desigualdad en mortalidad perinatal entre las comunas de la Provincia de Santiago. Rev Chil Obstet Ginecol 2004; 69(2): 112-17.

5. Donoso E. Desigualdad en mortalidad infantil entre las comunas de la Provincia de Santiago. Rev Méd Chil 2004; 132: 461-66.

6. Donoso E, Oyarzún E, Villarroel L. Mortalidad fetal tardía. Chile 1990-1996. Rev Chil Obstet Ginecol 1999; 64(1): 21-28.

7. Donoso E, Villarroel L. Mortalidad neonatal precoz. Chile 1991-1997. Rev Chil Obstet Ginecol 1999; 64(4): 286-91.

8. Elam-Evans LD, Strauss LT, Herndon J, Parker WY, Whitehead S, Berg,CJ. Abortion Surveillance - United State, 1999. En: Surveillance Summaries, November 29, 2002. MMWR; 51(№ SS-9): 1-32.

9. Ticona M, Huanco D. Mortalidad perinatal hospitalaria en el Perú: factores de riesgo. Rev ChIL OBStet GINECOL 2005; 70(5): 313-17.

\section{F E D E E R A T A}

Corrige filiación incompleta del Dr. José Solis C., de su manuscrito "Tratamiento adyuvante del cáncer cérvico uterino: factores de riesgo, indicaciones y tratamiento", publicado en: Rev ChiL OBstet GineCol 2005; 70(1): 41-8.

Dice: José Solis C. ${ }^{1}$

${ }^{1}$ Servicio de Oncología, Hospital Carlos Van Buren de Valparaíso.

Debe decir: José Solis C.1,2.

${ }^{1}$ Unidad de Radioterapia Oncológica, Servicio de Oncología, Hospital Carlos Van Buren, Valparaíso.

${ }^{2}$ Cátedra de Oncología, Escuela de Medicina, Facultad de Medicina, Universidad de Valparaíso. 